\title{
Field Compressing Magnetothermal Instability in Laser Plasmas
}

\author{
J. J. Bissell, C. P. Ridgers and R. J. Kingham \\ The Blackett Laboratory, Imperial College London, SW7 2BZ, England
}

(Dated: August 3, 2010)

\begin{abstract}
The mechanism for a new instability in magnetized plasmas is presented and a dispersion relation derived. Unstable behaviour is shown to result purely from transport processes - feedback between the Nernst effect and the Righi-Leduc heat-flow phenomena in particular - neither hydrodynamic motion nor density gradients are required. Calculations based on a recent nanosecond laser gas-jet experiment [1] predict growth of magnetic field and temperature perturbations with typical wavelengths of order $50 \mu \mathrm{m}$ and characteristic growth times of $\sim 0.1 \mathrm{~ns}$. The instability yields propagating magneto-thermal waves whose direction depends on the magnitude of the Hall Parameter.
\end{abstract}

PACS numbers: 52.25.Fi, 52.25.Xz, 52.35.-g

The existence of large self-generated magnetic fields in laser-produced plasmas $(\sim 100 \mathrm{~T})$ has long been known $[2,3]$. These fields can significantly affect the distribution of thermal energy in plasma targets by suppressing the cross-field thermal conductivity [4]. In recent years several experiments have been designed to assess their impact on inertial confinement fusion (ICF) schemes [5] and to study more general magnetic phenomena in laser plasmas, such as magnetic reconnection [6] and instability [7]. In addition, there has been increased discussion of the possible uses for applied magnetic fields in the suppression of non-local transport [1], control of plasma density channels [8], wakefield acceleration [9] and magnetized target fusion (MTF) schemes [10].

In this letter we report a new instability shown computationally to impact on magnetized plasmas, though it may also take effect in the presence of self-generated fields. The instability compresses the magnetic field and distorts thermal energy profiles by concentrating the heat-flow (see figure 1), and may be important when a high degree of symmetry or control of heat transport is needed, or where uniform fields are applied for a specific purpose, such as those cases mentioned above [1, 8-10].

Feedback is driven solely by classical (Braginskii) transport processes [4]: specifically the interaction of the Nernst effect, which describes advection of magnetic field with heat-flow down temperature gradients $\mathbf{q}_{\perp}$ and with velocity $\mathbf{v}_{N} \approx 2 \mathbf{q}_{\perp} / 5 P_{e}$, where $P_{e}$ is the isotropic pressure [11]; and the Righi-Leduc heat-flow, the cross-field thermal-flux 'bent' by magnetic fields acting on negatively charged heat-carrying electrons. Consequently we require only the presence of temperature gradients $\nabla T_{e}$ perpendicular to an existing magnetic field for instability. Gradients in electron number density $n_{e}$ are not needed (i.e. $\nabla n_{e}=0$, precluding $\nabla T_{e} \times \nabla n_{e}$ field generation), nor hydrodynamic motion or anisotropic pressure. Thus, what we see is distinct from instabilities existing in the literature: such as those of Tidman-Shanny [12-14], for which $\nabla T_{e} \times \nabla n_{e}$ is necessarily non-zero; Weibel [15], where magnetic fields are not essential; Haines [16, 17], which does not require either Righi-Leduc heat-flow or the Nernst effect; and Davies [18], where unstable filamentation arises from plasma motion.
In our case, terms responsible for growth go as $k^{\frac{3}{2}}$, where $k$ is the wavenumber of a perturbation (not the more usual $k$ ), yielding traveling waves rather than purely growing perturbations. These, however, differ from the thermal-magnetic waves described by Pert [19] who neglected the Nernst effect.

We present an analytical theory of the instability alongside results from numerical simulation in the context of an experimental arrangement which uses applied magnetic fields; specifically the conditions of Froula et al. [1], in kinetic modeling of which it was first observed [20]. In this experiment, designed to measure the suppression of non-local heat transport by magnetic fields, a nitrogen gas-jet (atomic number $\mathrm{Z}=7$ ), with electron number density $n_{e}=1.5 \times 10^{19} \mathrm{~cm}^{-3}$ and initial temperature $T_{e}=20 \mathrm{eV}$, was subject to inverse bremsstrahlung heating for $1 \mathrm{~ns}$ by a long-pulse laser of wavelength $1054 \mathrm{~nm}$ and intensity $6.3 \times 10^{14} \mathrm{Wcm}^{-2}$. Uniform magnetic fields of strengths up to $12 \mathrm{~T}$ were imposed parallel to the laserheating beam and the radial heat-flow inferred. These parameters should be assumed throughout.

Though the instability was originally observed in kinetic simulations [20], no theory was derived and to this end a classical transport model better elucidates the physics of the problem. Data presented here is thus taken primarily from our classical transport code CTC. Where appropriate, however, we present results from our kinetic code IMPACT [21] and from CTC+: a version of CTC which includes hydrodynamic motion. For the conditions considered here, simulation using CTC+ reveals that neither hydrodynamics nor density gradients impact heavily on the the instability (see figure 2), so that both effects are neglected in the theory at this stage.

For consistency with Froula et al. [1], we focus on a two-dimensional cross-section through a plasma perpendicular to both the applied magnetic field and the laser-heating beam. However, for simplicity we consider an $x-y$, rather than $r-\theta$ geometry, with a laser-heating strip resulting from a heating operator $\dot{U}_{L}(x)$ in place of a circular laser spot. We thus suppose a plasma with principal temperature and magnetic field gradients along the $x$-axis of the system only. The magnetic field is applied parallel to the $z$-axis, i.e. $\mathbf{B}=B \hat{\mathbf{z}}$, where $B=|\mathbf{B}|$, 
so that plasma scalar quantities $f$ and vector quantities $\mathbf{A}$ are such that $\mathbf{B} \cdot \nabla f=\mathbf{B} \cdot \mathbf{A}=0$. Snapshots of the instability in this geometry are shown in figure 1 .
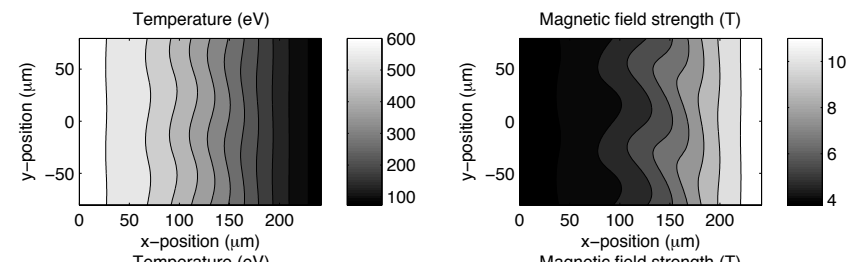
Temperature $(\mathrm{eV})$

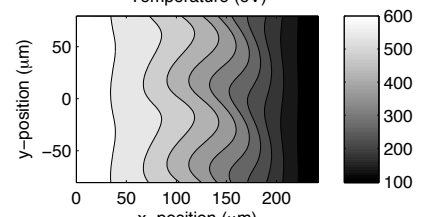
x-position (um)
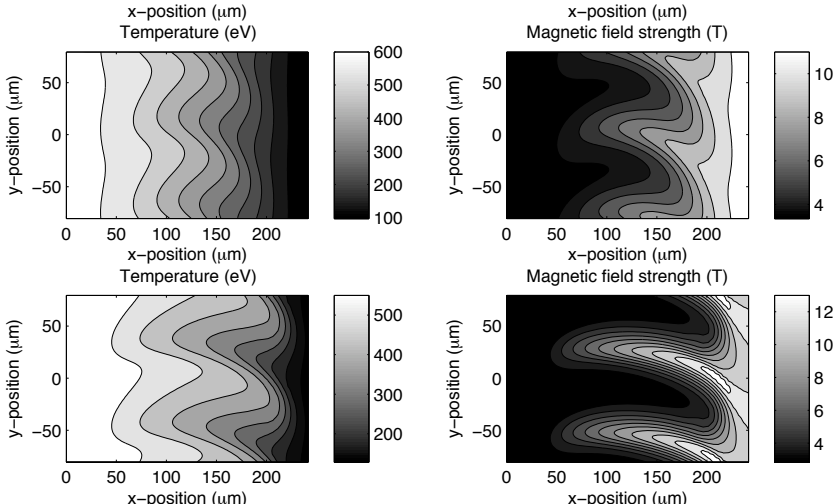

FIG. 1: Snapshots of the instability taken at 700ps (top), 800ps (centre) and 900ps (bottom), from CTC simulations of the experiment of Froula et al. [1] for the case of an $8 \mathrm{~T}$ field given a $1 \%$ perturbation at the laser 'switch-on' time.

Our transport code incorporates the full Braginskii model with corrected coefficients [4, 22, 23]. However, the theory presented here includes just the most important damping terms, resistive and thermal diffusion, alongside the main feedback terms describing the Nernst effect and Righi-Leduc heat-flow. The Ettingshausen term, which helps to mitigate diffusive effects, is also retained. In this way, using Braginskii's expression for the electric field $\mathbf{E}$ and Ampère's Law to write the current as $\mathbf{j}=(\nabla \times \mathbf{B}) / \mu_{0}$, and by neglecting hydrodynamics and density gradients $\left(\nabla n_{e}=0\right)$, the induction equation is:

$$
\begin{aligned}
\frac{\partial \mathbf{B}}{\partial t} & =-\nabla \times \mathbf{E} \\
& =-\nabla \times\left(\frac{m_{e} \alpha_{\perp}}{e^{2} n_{e} c_{B} \tau_{T} \mu_{0}} \nabla \times \mathbf{B}-\frac{\beta_{\wedge}}{e} \hat{\mathbf{z}} \times \nabla T_{e}\right) .
\end{aligned}
$$

Similarly, by neglecting Ohmic heating, using Braginskii's form for the heat-flow $\mathbf{q}$, and with a laser-heating operator $\dot{U}_{L}(x)$, the thermal-energy continuity equation of our reduced model becomes:

$$
\begin{aligned}
& \frac{3}{2} n_{e} \frac{\partial T_{e}}{\partial t}=-\nabla \cdot \mathbf{q}+\dot{U}_{L}=\nabla \cdot\left(\frac{T_{e}}{e \mu_{0}} \psi_{\wedge} \hat{\mathbf{z}} \times(\nabla \times \mathbf{B})\right) \\
& +\nabla \cdot\left(\frac{n_{e} c_{B} \tau_{T} T_{e}}{m_{e}}\left[\kappa_{\perp} \nabla T_{e}+\kappa_{\wedge} \hat{\mathbf{z}} \times \nabla T_{e}\right]\right)+\dot{U}_{L} .
\end{aligned}
$$

In these equations $e$ is the electronic charge; $c_{B}=$ $3 \sqrt{\pi} / 4$ is a dimensionless constant; and the thermal collision time $\tau_{T}=4 \pi v_{T}^{3} / n_{i}\left[Z e^{2} / \epsilon_{0} m_{e}\right]^{2} \ln \Lambda_{e i}$ is defined by the thermal velocity $v_{T}=\left(2 T_{e} / m_{e}\right)^{\frac{1}{2}}$, the ion number density $n_{i} \approx n_{e} / Z$ and the Coulomb logarithm $\ln \Lambda_{e i} \approx 8$. The transport coefficients - the resistivity $\alpha_{\perp}$, thermal conductivity $\kappa_{\perp}$, Nernst, Ettingshausen and Righi-Leduc terms, $\beta_{\wedge}, \psi_{\wedge}$ and $\kappa_{\wedge}$ respectively - are dimensionless functions of the atomic number $Z$ and Hall parameter $\chi=\omega_{g} c_{B} \tau_{T}$ only, where $\omega_{g}=e B / m_{e}$ is the electron gyro-frequency. These are calculated using polynomial fits in the Lorentz approximation [22, 23].

Taking zeroth order solutions to equations (1) and (2) of the form $B=B_{0}(x, t)$ and $T_{e}=T_{0}(x, t)$, we add wavelike perturbations with wavenumber $k$ and frequency $\omega$ at an (anticlockwise) angle $\theta$ to the $x$-axis. In this way we have $T_{e}=T_{0}+\delta T$ and $B=B_{0}+\delta B$, where $\delta T=$ $\delta T^{\prime} \exp i\left(k_{x} x+k_{y} y-\omega t\right), \delta B=\delta B^{\prime} \exp i\left(k_{x} x+k_{y} y-\omega t\right)$, $k_{x}=k \cos \theta, k_{y}=k \sin \theta$, and $\delta T^{\prime}$ and $\delta B^{\prime}$ are complex. Hence, by defining temperature and magnetic field scale lengths $L_{T}=T_{0} /\left(\partial T_{0} / \partial x\right)$ and $L_{B}=B_{0} /\left(\partial B_{0} / \partial x\right)$ respectively, and assuming $\left|k L_{T, B}\right| \gg 1$ and $\left|\nabla\left(1 / L_{T, B}\right)\right| \lesssim$ $1 / L_{T, B}^{2}$, the first order forms of equations (1) and $(2)$ yield a quadratic in $\omega$ and the dispersion relation:

$$
\begin{gathered}
\omega_{ \pm}=\frac{1}{2}\left\{s_{B} k-\left(d_{R}+d_{T}\right) i k^{2}\right\} \pm \frac{1}{2}\left\{s_{B}^{2} k^{2}\right. \\
\left.+\left[s_{P}+2 s_{B}\left(d_{R}-d_{T}\right)\right] i k^{3}-\left[\left(d_{R}-d_{T}\right)^{2}+s_{E}\right] k^{4}\right\}^{\frac{1}{2}}
\end{gathered}
$$

Here the additional $d$ and $s$ coefficients are defined in terms of the mean free path $\lambda_{T}=\tau_{T} v_{T}$ and the skin $\operatorname{depth} \delta=c / \omega_{p e}$, where $c$ is the speed of light and $\omega_{p e}=$ $\left(e^{2} n_{e} / m_{e} \epsilon_{0}\right)^{\frac{1}{2}}$ is the plasma frequency:

$$
s_{P}=\frac{2 \beta_{\wedge} c_{B}^{2} \lambda_{T}^{4}}{3 L_{T} \tau_{T}^{2}} \frac{\partial \kappa_{\wedge}}{\partial \chi} \sin \theta, \quad s_{B}=\frac{c_{B} \chi \lambda_{T}^{2}}{3 L_{B} \tau_{T}} \frac{\partial \kappa_{\wedge}}{\partial \chi} \sin \theta
$$

$s_{E}=\frac{4 \lambda_{T}^{2} \delta^{2}}{3 \tau_{T}^{2}} \beta_{\wedge} \psi_{\wedge}, \quad d_{R}=\frac{\alpha_{\perp} \delta^{2}}{c_{B} \tau_{T}} \quad$ and $\quad d_{T}=\frac{c_{B} \kappa_{\perp} \lambda_{T}^{2}}{3 \tau_{T}}$.

Note that $d_{R}$ and $d_{T}$ represent the coefficients for resistive and thermal diffusion respectively. The solution for $\omega_{+}$yeilds unstable modes for a range of $k$ up to a cut-off $k_{c}$ with growth rates given by $\Im\left\{\omega_{+}\right\}$(see figure $2)$, though the local approximation subjects us to the additional restriction $\left|1 / L_{T, B}\right| \ll|k| \ll 1 / \lambda_{T}$.

Perturbations grow primarily as a result of interplay between the Nernst effect and the Righi-Leduc heat-flow in the $s_{P}$ source term in equation (3), yielding growth that goes as $k^{\frac{3}{2}}$; while the main damping terms in $d_{R}$ and $d_{T}$ are proportional to $k^{2}$, giving us the form of the dispersion curve in figure 2 (the term in $s_{B}$ also contributes as a source, but is not essential). The angular dependence of $s_{B}$ and $s_{P}$ means that a $y$-component to the perturbation is needed for instability. In our simulations, and from henceforth in this letter, we take $\theta=\pi / 2$, i.e. $\sin \theta=1$. Feedback between the Nernst and Ettingshausen effects, which goes like $k^{2}$ and is accounted for by $s_{E}$, acts to reduce the impact of diffusion, but cannot 
itself drive instability due to its equivalent power in $k$. Thus, the instability is perhaps best understood by assessing the phenomena in the principal source term $s_{P}$.

The effect of a temperature perturbation on an unperturbed magnetic field $\left(B=B_{0}\right)$ may be considered by examining the first order correction due to the Nernst term in the induction equation:

$$
\left(\frac{\partial B}{\partial t}\right)_{\beta_{\wedge}}^{\mathrm{O}(1)}=\frac{\beta_{\wedge}}{e} k^{2} \delta T e^{i \pi} .
$$

Hence, a magnetic field perturbation is induced in antiphase. Physically this is a result of the compressional aspect of Nernst advection. The Nernst velocity - the velocity of advection - is proportional to $\partial T_{e} / \partial y$, so that magnetic field is compressed in the troughs of the temperature perturbation and rarefacted at the peaks.

Similarly, we consider the impact of a magnetic field perturbation on an unperturbed temperature profile $\left(\partial T_{0} / \partial x<0\right)$ using the first order correction due to the Righi-Leduc term in the energy continuity equation:

$$
\left(\frac{\partial T}{\partial t}\right)_{\kappa_{\curlywedge}}^{\mathrm{O}(1)} \propto \frac{\partial T_{0}}{\partial x} \frac{\partial \kappa_{\wedge}}{\partial \chi} k \delta B e^{i \frac{\pi}{2}}
$$

For $\chi$ greater than about $10^{-1}$ we have $\partial \kappa_{\wedge} / \partial \chi<0$, in which case the magnetic field perturbation will induce a temperature perturbation that leads by $\pi / 2$. This is due to the dependence of $\kappa_{\wedge}$ on $\chi$, which is itself directly proportional to $B$. Since $\partial \kappa_{\wedge} / \partial \chi<0$, regions of higher magnetic field strength have a lower Righi-Leduc heatflow, so that heat is transported away from these regions more slowly than those of lower $B$. Thus, as we move along the positive $y$-axis, thermal energy is built up in places where heat-flow goes from high to low and removed from places where it goes from low to high. The reverse is found if $\chi$ is less than about $10^{-1}$, where $\partial \kappa_{\wedge} / \partial \chi>0$.

The two stages of this feedback process result in induced perturbations which have different phases. Magnetic field perturbations will tend to 'push' temperature perturbations towards a phase difference of $\pm \pi / 2$ ( $\operatorname{sign}$ identical to that of $\left.\left[\partial T_{0} / \partial x\right]\left[\partial \kappa_{\wedge} / \partial \chi\right]\right)$, while temperature perturbations 'pull' magnetic field perturbations towards a phase of $\pi$. The net result of this 'push-pull' interaction is that perturbations propagate as waves with a phase difference of $\phi \approx 3 \pi / 4$ and in the direction $\pm \hat{\mathbf{y}}$ (again, sign identical to that of $\left[\partial T_{0} / \partial x\right]\left[\partial \kappa_{\wedge} / \partial \chi\right]$ ).

The dependence of the $d$ and $s$ coefficients on $T_{0}(x, t)$ and $B_{0}(x, t)$ means that the growth rate varies both temporally and spatially. Evaluation of the dispersion relation is thus limited to a particular plasma cross-section $x$ and based on a given snapshot of the bulk profile. When using profiles taken from computational simulation of the experiment of Froula et al. [1], the theoretical model and growth rates measured from full heating simulations show good agreement (see figure 2).
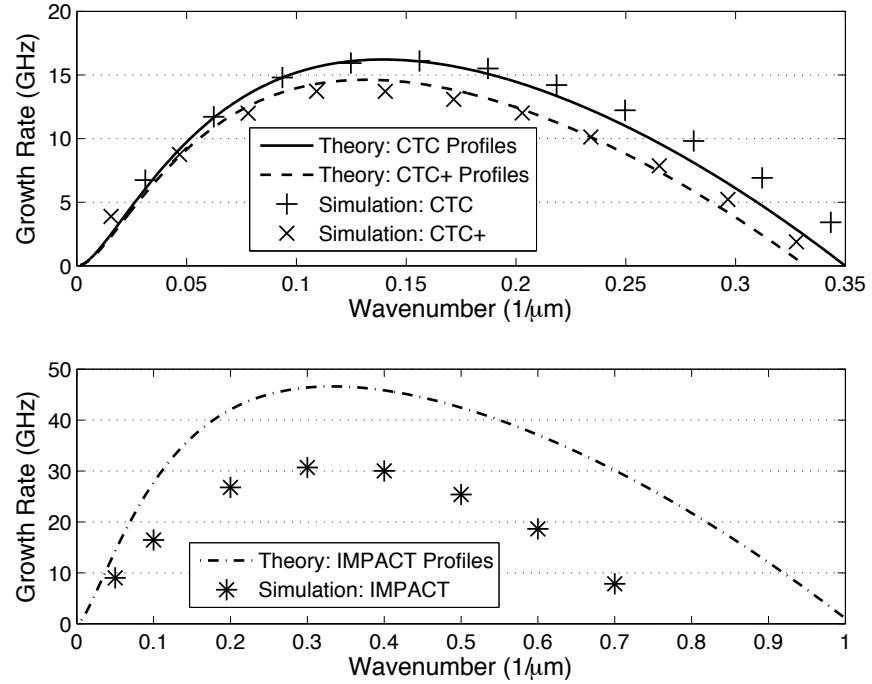

FIG. 2: The theoretical dispersion relation of our reduced model - for a $6 \mathrm{~T}$ magnetized plasma at a distance $x \approx 120 \mu \mathrm{m}$ from the laser strip centre after 500ps of heating - based on one-dimensional profiles taken from CTC (top plot, solid line), CTC+ (top plot, dashed line) and IMPACT (bottom plot). These may be compared with growth-rates measured from full two-dimensional simulations.

As indicated in figure 2, for the conditions considered here hydrodynamics does not significantly affect the instability. Non-local effects, on the other hand, which are relevant to the experiment of Froula et al. [1], do reduce the predictive power of the theory by modifying the transport coefficients; though the physical mechanism of the instability remains the same. However, simulation using IMPACT (see figure 2) shows that this reduction is not dramatic: the peak wavenumber is effectively unchanged, while the cut-off wavenumber and peak growth rate agree to within $\sim 35 \%$. Indeed, it is noteworthy that though growth rates measured from kinetic simulations are lower than those predicted by the theory, the different bulk profiles mean that rates are approximately twice those taken from CTC and CTC+.

The cut-off wavenumber for unstable modes $k_{c}$, calculated by solving $\Im\left\{\omega_{+}\right\}=0$, may be expressed in terms of the dimensionless parameters $\chi$ and $\Lambda=\left(\lambda_{T} / \delta\right)$ :

$$
\begin{gathered}
k_{c} L_{T}=\frac{c_{B}}{3} \frac{\partial \kappa_{\curlywedge}}{\partial \chi}\left(\frac{3}{\alpha_{\perp} \kappa_{\perp}-\beta_{\wedge} \psi_{\wedge}}\right)^{\frac{1}{2}}\left(\frac{\beta_{\wedge}}{2}-\frac{\kappa_{\perp} \chi}{3} \frac{L_{T}}{L_{B}}\right)^{\frac{1}{2}} \\
\times\left(\alpha_{\perp} \chi \frac{L_{T}}{L_{B}}+\frac{\beta_{\wedge} c_{B}^{2}}{2} \Lambda^{2}\right)^{\frac{1}{2}}\left(\frac{\alpha_{\perp}}{c_{B} \Lambda^{2}}+\frac{c_{B} \kappa_{\perp}}{3}\right)^{-1} .
\end{gathered}
$$

This equation makes clear the necessity of both the Nernst effect and Righi-Leduc heat-flow in driving instability: without the former $\left(\beta_{\wedge}=0\right), k_{c}$ becomes imaginary, and without the latter $\left(\partial \kappa_{\wedge} / \partial \chi=0\right), k_{c}$ is zero. It also demonstrates how feedback between the Nernst and Ettingshausen effects reduces the impact of the diffusive term in $\alpha_{\perp} \kappa_{\perp}$ - thus extending the range of instability. 
No equivalent expression for the peak wavenumber $k_{M}$ exists, however, for the experiment of Froula et al. [1] we find $\left|s_{B}^{2}-\left[\left(d_{R}-d_{T}\right)^{2}+s_{E}\right] k_{M}^{2}\right| \approx\left|\left[s_{P}+2\left(d_{R}-d_{T}\right) s_{B}\right] k_{M}\right|$, so that the peak growth rate $\gamma_{M}$ and wavenumber $k_{M}$ may be approximated by $\gamma_{M}^{\prime}$ and $k_{M}^{\prime}$ respectively, where:

$$
\begin{gathered}
\gamma_{M}^{\prime}=\frac{1}{2}\left[s_{P}+2\left(d_{R}-d_{T}\right) s_{B}\right]^{\frac{1}{2}} k_{M}^{\prime}{ }^{\frac{3}{2}}-\frac{1}{2}\left(d_{R}+d_{T}\right) k_{M}^{\prime 2} \\
\text { and } \quad k_{M}^{\prime}=\frac{9}{16}\left[s_{P}+2\left(d_{R}-d_{T}\right) s_{B}\right]\left[d_{R}+d_{T}\right]^{-2}
\end{gathered}
$$

More precise values for both $\gamma_{M}$ and $k_{M}$ may be found computationally under the assumption $L_{T} \approx-L_{B}$. Using $\Lambda=20$ (characteristic for the experiment of Froula et al. [1]) and by comparing these values with $\gamma_{M}^{\prime}$ and $k_{M}^{\prime}$, we find that the approximate expressions agree to within a factor of five for $10^{-2}<\chi<10^{2}$.

To enable more qualitative discussion, we simplify further by assuming no magnetic field gradients $\left(L_{B} \rightarrow\right.$ $\infty)$, so that $s_{B}=0$, take $\Lambda \gg \max \{1, \chi\}$, for which $d_{T} \gg d_{R}$, and combine equations (7) and (8) to express the peak growth rate as $\gamma_{M}^{\prime}=(3 / 8)^{3} s_{P}^{2} d_{R}^{-3}$, i.e. $\gamma_{M}^{\prime} \tau_{T} \approx\left[\left(\beta_{\wedge} \lambda_{T} / L_{T}\right)\left(\partial \kappa_{\wedge} / \partial \chi\right)\right]^{2} \kappa_{\perp}^{-3}$. Writing $\gamma_{M}^{\prime}$ in this way emphasizes the importance of steep temperature gradients, through $1 / L_{T}^{2}$, and of intermediate Hall Parameter, to allow significant $\beta_{\wedge}^{2}\left(\partial \kappa_{\wedge} / \partial \chi\right)^{2} / \kappa_{\perp}^{3}$. More specifically, we require $\chi$ in the region of $10^{-2}$ to $10^{2}$, but avoiding $\chi \sim 10^{-1}$ where $\partial \kappa_{\wedge} / \partial \chi \sim 0$. Furthermore, the dimensionless form indicates relevance to other plasmas in self-similar regimes.

Under the conditions considered in this letter, the instability has growth rates of order $10 \mathrm{GHz}$, optimal wavelengths of $\sim 50 \mu \mathrm{m}$ and can significantly disrupt magnetic field and temperature profiles over nanosecond timescales when compared to stable heating simulations. By concentrating the heat-flow in regions where the magnetic field is rarefacted, the instability enhances the spread of thermal energy (see figure 3).

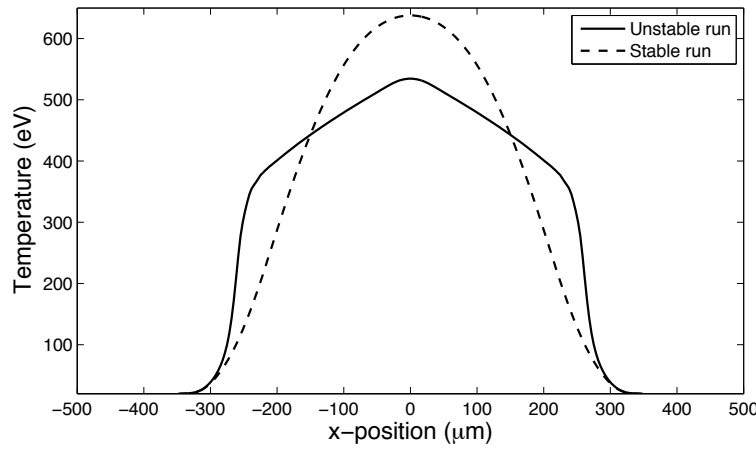

FIG. 3: A comparison between the spread of thermal energy when the instability is active (solid line) and when it is not (dashed line) after $1 \mathrm{~ns}$ of laser heating for the case of an $8 \mathrm{~T}$ applied field (c.f. figure 1). The data, taken from CTC, is averaged in $y$ for each $x$ cross-section.

To conclude, we have derived the linear theory for a new plasma instability in magnetized plasmas, which predicts propagating waves with growing amplitude for a range of wavenumbers, and have shown that this theory compares well with simulation. Uniquely, the instability results solely from feedback between collisional transport processes, principally the Nernst effect and Righi-Leduc heat-flow; though further investigation of the effects of hydrodynamics and non-local heat-flow are key areas of future work. The instability is likely to be important for ICF (particularly hohlraum gas-fill conditions which are similar to those considered here) and most likely MTF. Furthermore, its existence highlights the necessity of including the Nernst effect and Righi-Leduc heat-flow in magneto-hydrodynamic models for any plasma of intermediate magnetization.

We would like to acknowledge useful contributions from Dr A. G. R. Thomas. This work was funded by the UK Engineering and Physical Sciences Research Council (Grant No. EP/P502500/1).
[1] D. H. Froula et al., Phys. Rev. Lett. 98, 135001 (2007).

[2] J. A. Stamper et al., Phys. Rev. Lett. 26, 1012 (1971).

[3] A. Raven, O. Willi, and P. T. Rumsby, Phys. Rev. Lett. 41, 554 (1978)

[4] S. I. Braginskii, Reviews of Plasma Physics 1, p. 205 (1965).

[5] P. M. Nilson et al., Phys. Rev. Lett. 97, 255001 (2006).

[6] C. K. Li et al., Phys. Rev. Lett. 99, 055001 (2007).

[7] C. K. Li et al., Phys. Rev. Lett. 99, 015001 (2007).

[8] D. H. Froula et al., Plas. Phys. Conrol. Fusion 51, 024009 (2009).

[9] T. Hosokai et al., Phys. Rev. Lett. 97, 075004 (2006).

[10] I. R. Lindemuth et al., Phys. Rev. Lett. 75, 1953 (1995).

[11] A. Nishiguchi, T. Yabe, and M. G. Haines, Phys. Fluids 28, 3683 (1985).

[12] D. Tidman and R. Shanny, Phys. Fluids 17, 1207 (1974).

[13] M. Ogasawara, A. Hirao, and H. Ohkubo, J. Phys. Soc.
Jpn 49, 322 (1980).

[14] A. Hirao, J. Phys. Soc. Jpn 50, 668 (1981).

[15] E. S. Weibel, Phys. Rev. Lett. 2, 83 (1959).

[16] M. G. Haines, J. Plas. Phys. 12, 1 (1974).

[17] M. G. Haines, Phys. Rev. Lett. 47, 917 (1981).

[18] J. R. Davies et al., Plas. Phys. Conrol. Fusion 51, 035013 (2009).

[19] G. J. Pert, J. Plas. Phys. 18, 227 (1977).

[20] C. P. Ridgers, R. J. Kingham, and A. G. R. Thomas, Phys. Rev. Lett 100, 075003 (2008).

[21] R. J. Kingham and A. R. Bell, J. Comp. Phys. 194, 1 (2004).

[22] E. M. Epperlein and M. G. Haines, Phys. Fluids 29, p. 1029 (1986).

[23] C. P. Ridgers et al., Phys. Plas. 15, 092311 (2008). 